\title{
Serum ErbB2 concentration positively correlated to the glycemic variations in newly diagnosed Type 2 diabetic patients
}

\section{Yan Huang}

Yancheng No.1 People's Hospital

\section{Xia Han}

Zhimaying Community Health Center

Feng-fei Li

Nanjing First Hospital https://orcid.org/0000-0002-6127-6604

Yan-mei Liu

Yancheng No.1 People's Hospital

Zelong Liu

Icahn School of Medicine at Mount Sinai

Yu-qing She ( $\nabla$ susan710316@163.com )

\section{Research}

Keywords: Type 2 Diabetes, glycemic control, ErbB2

Posted Date: February 26th, 2020

DOI: https://doi.org/10.21203/rs.2.24651/v1

License: (c) (i) This work is licensed under a Creative Commons Attribution 4.0 International License.

Read Full License

Version of Record: A version of this preprint was published at Scientific Reports on March 23rd, 2022. See the published version at https://doi.org/10.1038/s41598-022-07549-x. 


\section{Abstract}

\section{Background}

Accumulated evidences indicate that elevated levels of circulating ErbB2 are closely associated with increased incidence of diabetes. However, the relationship between ErbB2 concentration and glycemic variations (GV) in type 2 diabetic (T2D) patients remains elucidated. The aim of this study was to assess whether there is an association between serum ErbB2 concentration and GV in newly diagnosed T2D patients.

Methods

This was a three-center, and observational study. Between April 2019 and July 2019, a total of 106 newly diagnosed T2D patients were recruited. All recruited subjects were admitted as inpatients and received anti-diabetes agents free during the study period. At baseline, fasting serum was collected for ErbB2 measurement and all recruited patients were subjected a prospective CGM for at least 3 days. The primary endpoint was the relationships between ErbB2 concentrations and GV in T2D patients.

Results

Data of a total of 95 subjects who met the inclusion criteria were analyzed at the endpoint. Subjects were divided into quartiles according to their serum ErbB2 concentrations. We observed that subjects with an elevated level of ErbB2 had a higher value of GV in terms of mean amplitude of glucose excursion (MAGE), standard deviation of mean glucose (SDMG), and the coefficient of variation (CV\%) than those with lower levels (all $P<0.05$ ). Multiple linear regression analyzes after adjusting for confounder factors indicate that serum ErbB2 levels were significantly positively correlated with the MAGE $(\beta=0.664, t=7.218$, $P<0.01), S D(\beta=0.469, t=5.125, P<0.01)$ and $C V \%(\beta=0.337, t=4.442, P<0.01)$, respectively.

Conclusion

Serum ErbB2 concentration was positively correlated to the glycemic variations in newly diagnosed T2D patients. Our data indicating that ErbB2 may be a potential treatment target for diabetic patients for improvement in glycemic control.

Keywords

Type 2 Diabetes, glycemic control, ErbB2

\section{Background}

The ErbB2/HER2 oncoprotein, a member of receptor of tyrosine kinases, is a transmembrane protein, which consists of the internal tyrosine residues, the transmembrane portion, and the external extracellular domain $(E C D)^{1,2}$. Homo- or heterodimerization leads to ErbB2 activation, which initiates a multiple of 
downstream signaling pathways regulating cell proliferation, apoptosis, and differentiation ${ }^{3}$. Various studies have shown that serum ErbB2/HER2 concentrations elevation is a specific marker for variety of cancers, such as breast cancers ${ }^{4,5}$, ovarian, bladder, salivary gland, endometrial, pancreatic, and nonsmall-cell lung cancer ${ }^{6}$. Studies also indicate that tumor patients with ErbB2 amplification or overexpression have an enhanced risk of poorer prognosis ${ }^{7}$.

Epidemiological studies reveal that diabetic patients have a potential risk of increase in incidence of cancers $^{8}$, and pre-diagnosed diabetic patients with tumors had an increased in all-cause and cancer related mortality compared with those normal glucose metabolism individuals ${ }^{9,10}$. The underline biological mechanisms between the two heterogeneous, chronic and progressive diseases may partly be the reason by the two conditions shared the same metabolism risk factors such as those of aging, obesity, and $\operatorname{diet}^{8}$. Furthermore, the links between diabetes and cancer are then strengthen by the activation of the insulin receptor, insulin-like growth factor receptors (IGFR) ${ }^{11}$ and ErbB2 ${ }^{12}$. Studies demonstrated that ErbB2 play a role in controlling lipid metabolism ${ }^{13}$ and involved in impaired glucose metabolism ${ }^{14}$. Fueling the notion that ErbB2 may be the bridge which connection of diabetes and cancers, in which anti-diabetic agents may be an therapy somewhat beneficial for cancer subjects ${ }^{15}$. This hypothesis was confirmed by an in vitro study reported that glucose-lowering agents metformin has anticancer effect by mTOR/p70S6K1-sensed ROS downregulating HER2 expression ${ }^{12}$.

Very recently, a Population-Based Cohort Study confirmed that elevated levels of ErbB2 are associated with increased incidence of diabetes ${ }^{16}$. However, the effect of serum ErbB2 concentrations on glycemic variation in type 2 diabetic (T2D) patients has not been elucidated.

We therefore performed a three-center, and observational study using continuous glucose monitoring (CGM) to assess the relationship between ErbB2 concentrations and glucose variations in T2D patients.

\section{Methods}

This was a three-center, and observational study. Between April 2019 and July 2019, a total of 106 newly diagnosed T2D patients were recruited in Department of Endocrinology, Yancheng No. 1 People's Hospital, The Fourth Affiliated Hospital of Nantong University, China, Department of Endocrinology, Nanjing Pukou Central Hospital, Pukou Branch Hospital of Jiangsu Provence Hospital, China, and Zhimaying Community Health Service Center, Qinhuai District, Nanjing, China. The inclusion criteria were:

1) Newly diagnosed T2D patients with random blood glucose less than $22.2 \mathrm{mmol} / \mathrm{L} ; 2$ ) with $\mathrm{HbA}_{1 \mathrm{c}}<$ $12.0 \%$; 3) aged between 18 and 80 years; 4) BMI 21 to $35 \mathrm{~kg} / \mathrm{m}^{2}$. Patients were excluded if they had the admission blood glucose higher than $22.2 \mathrm{mmol} / \mathrm{L}$, with chronic kidney or liver disease, or if they were diagnosed as maturity onset diabetes in the young (MODY) ${ }^{17,18}$. The study was approved by the ethics committee of Yancheng No. 1 People's Hospital, The Fourth Affiliated Hospital of Nantong University, China. All patients gave written informed consent. The methods were conducted in accordance with the Declaration of Helsinki guidelines, including any relevant details. 
All recruited subjects were admitted as inpatients and received anti-diabetes agents free during the study period. Oral glucose tolerance tests (OGTTs) were performed at 0800 on day 1 after admission using $75 \mathrm{~g}$ glucose diluted in $100 \mathrm{ml}$ water, and serum samples for $\mathrm{HbA}_{1 \mathrm{c}}$, glucose, insulin and C-peptide measurement were obtained at 0,30 , and 120 min after glucose loading. Insulin concentration was measured by an insulin radioimmunoassay kit (Beijing Technology Company, Beijing, China). $\mathrm{HbA}_{1 \mathrm{c}}$ value was delivered by a DiaSTAT HbA $1 \mathrm{c}$ analyzer (Bio-Rad, Hercules, CA). C-peptide and glucose concentrations were measured centrally at the central laboratory in Yancheng No. 1 People's Hospital. Fasting ErbB2 concentrations were measured with a commercially available quantitative ELISA (Oncogene Science, Bayer Diagnostics) according to the manufacturer's protocol.

After the baseline data were collected, prospective CGM (Sof-sensor, CGMS-Gold, Medtronic Incorporated, Northridge, USA) was performed for 3 days, as described previously ${ }^{19,20}$. In briefly, the subcutaneous glucose sensor was embedded in abdomen on Day 1 approximately 1600 PM and was removed on day 4 at 1600 PM. During the CGM period, at least 4 finger-stick readings should be entered for calibration every day. After the sensors removed, CGM data were recorded by the investigators, as described previously ${ }^{19-21}$. All subjects were instructed to maintain moderate activity with serving breakfast, lunch and dinner at 0700,1100 and 1700 , consisting of carbohydrate, proteins and fats in $55 \%, 17 \%$ and $28 \%$ ratio, respectively.

The readings delivered from CGM was recorded by researchers. The 24-hr mean glucose concentration (MG), the standard deviation of MG (SDMG), the coefficient of variation (CV\%), the incremental area under the curve (AUC) of glucose concentrations above $10.0 \mathrm{mmol} / \mathrm{L}$ or the incremental area over the curve (AOC) less than $3.9 \mathrm{mmol} / \mathrm{L}$, the time in range (TIR) glucose levels vary from 3.9 to $10.0 \mathrm{mmol} / \mathrm{L}$, and the hourly MG were calculated by researchers. The mean amplitude of glucose excursion (MAGE) was calculated manually, as previously described ${ }^{19,20}$. In addition, $\beta$-cell function and insulin sensitivity was presented by the homoeostasis model assessment B (HOMA-B), HOMA-IR ${ }^{17,22}$ and the Matsuda index 23,24 , respectively.

The primary endpoint was the relationships between ErbB2 concentrations and glucose variations in T2D patients. The differences in 24-hr MG, SDMG, incremental AUC (glucose $>10.0 \mathrm{mmol} / \mathrm{L}$ ) and the incremental AOC (glucose $<3.9 \mathrm{mmol} / \mathrm{L}$ ), the TIR, hourly MG, $\beta$-cell function, and insulin sensitivity in patients with different ErbB2 concentrations were also analyzed.

\section{Statistical analysis}

The normal distribution data were presented as the means \pm SD. Statistical analysis was performed using SPSS software (version 17.0; SPSS, Inc., Chicago, IL). Shapiro-Wilk test was used to verify the distribution of data. A Chi-squared test was performed to compare the ratio differences between two groups. All repeated data were analyzed by a two-way ANOVA between groups, followed by Bonferroni-Dunn post hoc test. $P$ values were two-tailed with a significance level of $5 \%$. 


\section{Results}

\section{Baseline characteristics}

A total of 106 newly diagnosed T2D subjects were screened for eligibility between tween April 2019 and July 2019 at Department of Endocrinology, Yancheng No. 1 People's Hospital, The Fourth Affiliated Hospital of Nantong University, China, Department of Endocrinology, Pukou district Central Hospital, Jiangsu, China, and Zhimaying Community Health Service Center, Qinhuai District, Nanjing, China. All subjects fulfilled the study with eleven subjects were excluded from analyze: 5 with capillary glucose concentrations above $22.2 \mathrm{mmol} / \mathrm{L}$, and another 6 patients had readings of CGM missing for at least $10 \%$. A total of 95 newly diagnosed T2D subjects who met the inclusion criteria (53 men and 42 women, aged $55.1 \pm 7.6$ years, $\mathrm{BMI} 24.7 \pm 3.6 \mathrm{~kg} / \mathrm{m}^{2}$, and $\mathrm{HbA}_{1 \mathrm{c}}$ values $9.2 \pm 1.8 \%$ ) were analyzed at the endpoint. We observed that patients with newly diagnosed T2D had ErbB2 concentrations from $13.2 \pm 3.8$ to $6.3 \pm$ $2.2 \mathrm{ng} / \mathrm{ml}$.

\section{Glycemic variations in subjects with different ErbB2 concentrations}

We analyzed data of Day 1 to Day 3 of CGM at the endpoint, because of CGM has an infiltration phage at the beginning of monitoring period and a sensor expire phage at the ending of monitoring period, which might not be reliable according to the manufacture's instruction. In detail, all patients subjected CGM at $1600-1500$, yielding $751 \pm 76$ glucose readings per patient.

To observe whether patients with various ErbB2 concentrations had the same glycemic variations, subjects were assigned into quartiles according to their serum ErbB2 concentrations. The demographic data were comparable across the four groups as shown in Table 1. The different in glycemic variations were presented in Table 2. We observed that subjects with an elevated level of ErbB2 generally had a higher value of GV in terms of MAGE, SD, and CV\% (all P<0.05) than those with lower level (Table 2). 
Table 1

Baseline characteristics of subjects across 4 quartiles of ErbB2 concentrations

\begin{tabular}{|llllll|}
\hline Items & Quartile 1 & Quartile 2 & Quartile 3 & Quartile 4 & ANOVA p \\
\hline $\begin{array}{l}\text { Number } \\
\text { Male }\end{array}$ & 23 & 24 & 24 & 24 & 1 \\
\hline Age & $54.6 \pm 10.8$ & $52.1 \pm 12.5$ & $56.7 \pm 8.9$ & $53.2 \pm 12.0$ & 0.78 \\
\hline BMI & $23.3 \pm 5.6$ & $24.1 \pm 6.0$ & $25.0 \pm 8.4$ & $23.9 \pm 7.2$ & 0.81 \\
\hline HbA1c & $9.0 \pm 2.0$ & $9.5 \pm 4.6$ & $8.8 \pm 1.7$ & $9.3 \pm 2.5$ & 0.82 \\
\hline Waistline & $78.1 \pm 28.4$ & $74.6 \pm 20.1$ & $82.3 \pm 19.6$ & $75.8 \pm 23.0$ & 0.79 \\
\hline Waist-to-hip & $0.8 \pm 0.4$ & $0.8 \pm 0.2$ & $0.8 \pm 0.3$ & $0.8 \pm 0.2$ & 0.92 \\
\hline $\begin{array}{l}\text { Data were presented as means } \pm \text { SD. Age: (yrs); BMl: body mass index }\left(\mathrm{Kg} / \mathrm{m}^{2}\right), \mathrm{HbA} 1 \mathrm{c} \text { : Glycated } \\
\text { hemoglobin (\%); Waistline (cm); Waist-to-hip: Waist-to-hip ratio (\%). }\end{array}$ \\
\hline
\end{tabular}

Table 2

Glycemic profiles in recruited subjects across 4 quartiles of ErbB2 concentrations

\begin{tabular}{|c|c|c|c|c|c|}
\hline Items & Quartile 1 & Quartile 2 & Quartile 3 & Quartile 4 & ANOVA $p$ \\
\hline $\begin{array}{l}\text { Number } \\
\text { 24-hrs MG }\end{array}$ & $\begin{array}{l}23 \\
8.7 \pm 1.9\end{array}$ & $\begin{array}{l}24 \\
8.6 \pm 3.2\end{array}$ & $\begin{array}{l}24 \\
8.3 \pm 1.2\end{array}$ & $\begin{array}{l}24 \\
8.2 \pm 2.1\end{array}$ & ' 0.18 \\
\hline SDMG & $1.6 \pm 1.1$ & $1.9 \pm 1.8$ & $2.2 \pm 1.0$ & $2.6 \pm 1.3$ & 0.03 \\
\hline MAGE & $5.4 \pm 2.1$ & $5.7 \pm 1.2$ & $6.4 \pm 1.0$ & $7.1 \pm 2.6$ & 0.01 \\
\hline CV\% & $18.4 \pm 12.3$ & $22.7 \pm 20.5$ & $26.7 \pm 21.6$ & $31.7 \pm 21.6$ & 0.02 \\
\hline TIR & $72.1 \pm 28.0$ & $64.3 \pm 21.1$ & $42.9 \pm 31.4$ & $35.6 \pm 23.2$ & 0.00 \\
\hline$A U C>10.0$ & $0.3 \pm 0.4$ & $0.5 \pm 0.2$ & $0.6 \pm 0.3$ & $0.8 \pm 0.2$ & 0.00 \\
\hline$A \cup C<3.9$ & $0.3 \pm 0.0$ & $0.2 \pm 0.0$ & $0.0 \pm 0.0$ & $0.0 \pm 0.0$ & 0.23 \\
\hline \multicolumn{6}{|c|}{ 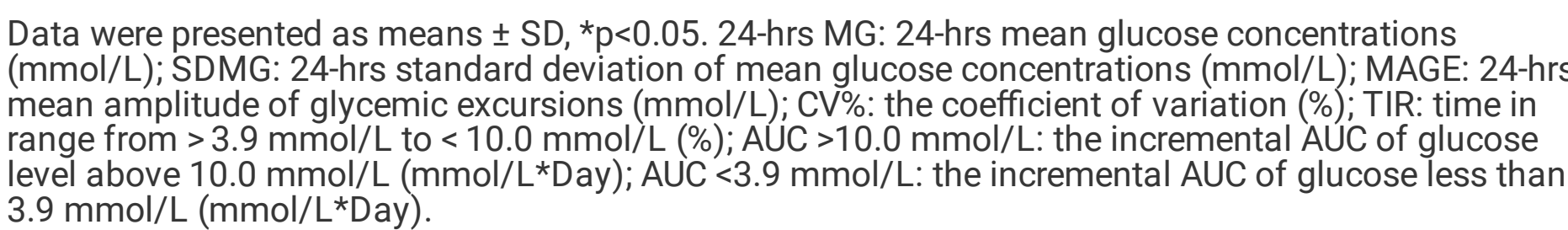 } \\
\hline
\end{tabular}

In the present study, our CGM data showed that the 24-hr SDMG, the MAGE, the CV\%, and the incremental area under the curve (AUC) of the glucose above $10 \mathrm{mmol} / \mathrm{L}$ were progressively and significantly amplified alongside ErbB2 levels values in newly diagnosed T2D patients. As expected, patients in the higher quartiles of ErbB2 levels had significantly decreased in TIR compared to those located in the lower quartiles (Table 2). 
In addition, we did not observe a statistically differences in the 24-hr MG and the incremental AOC of hypoglycemia across the quartiles in all recruited subjects. In addition, no hypoglycemic episode (defined as finger-stick glucose less than $3.9 \mathrm{mmol} / \mathrm{L}$, and/or symptomatic hypoglycemia) was reported during the study period. However, our CGM data demonstrated that 6 patients in the highest quartile experienced hypoglycemic episode (defined as CGM glucose reading $<3.9 \mathrm{mmol} / \mathrm{L}$ ), and 1 patient in the third quartile who experienced hypoglycemic episode, with the hypoglycemia durations ranging from $10 \mathrm{~min}$ to $60 \mathrm{~min}$. We did not observe hypoglycemic episode delivered by CGM in subjects in the other two quartiles.

\section{Relationships between ErbB2 concentration and glycemic variations}

A multiple linear regression analyzes were employed to assess the correlation between ErbB2 and MAGE. Our data indicated that only $\mathrm{HbA}_{1 \mathrm{c}}, \mathrm{BMI}$, ErbB2, FBG, MG, HOMA-IR and SDMG remained significant in the stepwise regression analysis. The standardized regression coefficients were $0.516(t=6.27, P<0.01)$, $0.496(t=6.112, P<0.01), 0.464(t=5.634, P<0.01), 0.433(t=5.535, P<0.01), 0.283(t=3.218, P<0.01)$, $-0.201(t=-3.482, P<0.05)$ and $0.175(t=2.387, P<0.05)$, respectively. After controlling for $\mathrm{HbA}_{1 c}, \mathrm{BMI}, \mathrm{FBG}$, MG, HOMA-IR and SDMG, the ErbB2 concentration was still significantly positively correlated with MAGE $0.664(t=7.218, P<0.01)$. Similarly, ErbB2 concentration was significantly positively correlated with SD ( $\beta$ $=0.469, t=5.125, P<0.01)$ and $C V \%(\beta=0.337, t=4.442, P<0.01)$.

\section{Relationships between ErbB2 concentration and glycemic variations}

We also observed the relationships between ErbB2 concentration and $\beta$-cell function/insulin sensitivity in patients with different ErbB2 values. Our data showed that patients with higher ErbB2 concentration exhibited an increase in HOMA-IR values and induction of Matsuda index (Table 3). However, there was no differences in HOMA-B values across the quartiles of ErbB2 concentrations. The multivariate analysis controlled for age and BMI to determine the relationships between ErbB2 concentration and $\beta$-cell function/insulin sensitivity. Our data showed that ErbB2 values only had a significantly negative correlation with HOMA-IR $(\beta=0.422, t=4.117, P<0.01)$ and Matsuda index $(\beta=0.317, t=2.885, P<0.05)$. We did not observe a statistically significant relationship between ErbB2 concentration and $\beta$-cell function. 
Table 3

The $\beta$-cell function and insulin sensitivity in recruited subjects across 4 quartiles of ErbB2 concentrations

\begin{tabular}{|llllll|}
\hline Items & Quartile 1 & Quartile 2 & Quartile 3 & Quartile 4 & ANOVA p \\
\hline $\begin{array}{l}\text { Number } \\
\text { C-peptide 0 }\end{array}$ & 23 & 24 & 24 & 24 & $/$ \\
\hline C-peptide 30 & $3.8 \pm 1.7$ & $3.2 \pm 0.5$ & $2.9 \pm 3.2$ & $2.8 \pm 2.7$ & 0.42 \\
\hline C-peptide 120 & $5.2 \pm 2.3$ & $5.4 \pm 0.8$ & $5.4 \pm 1.1$ & $5.1 \pm 2.7$ & 0.36 \\
\hline HOMA-B & $19.1 \pm 15.4$ & $20.3 \pm 21.0$ & $21.7 \pm 17.2$ & $18.9 \pm 16.6$ & 0.66 \\
\hline HOMA-IR & $2.1 \pm 2.3$ & $2.6 \pm 1.1$ & $3.4 \pm 1.0$ & $3.8 \pm 1.1$ & $0.04^{*}$ \\
\hline Mastuda Index & $116.8 \pm 81.5$ & $107.1 \pm 22.7$ & $96.3 \pm 12.8$ & $85.0 \pm 33.9$ & $0.03^{*}$ \\
\hline $\begin{array}{l}\text { Data were presented as means } \pm \text { SD, *p }<\text { 0.05. C-peptide 0: C-peptide 0 min (ng/mL); C-peptide 30: C- } \\
\text { peptide 30 min (ng/mL); C-peptide 120: C-peptide 120 min (ng/mL); HOMA-B: the homoeostasis } \\
\text { model assessment B; HOMA-IR: the homoeostasis model assessment IR. }\end{array}$ & & & \\
\hline
\end{tabular}

\section{Discussion}

In this observational study, our data indicating that newly diagnosed T2D patients with higher serum ErbB2 concentrations exhibited increase in glycemic variations regarding to MAGE, SD and CV\% compared to those of subjects with lower serum ErbB2 concentrations. Multivariate linear regression analyses on the whole study population revealed that serum ErbB2 concentration was positively correlated to the glycemic variations, and this association remained significant after adjusting for potential confounding factors such as those of age, sex, and BMI. Our data also showed that T2D patients with higher serum ErbB2 concentrations had an increase in insulin resistance in terms of HOMAIR and Matsuda index compared to those with lower ErbB2 concentrations.

It's well documented that ErbB2 is been widely investigated as an oncogenic marker and the predictor of cancer prognosis ${ }^{7}$. Because of exists a close association between metabolism and insulin resistance, which intriguing to explore a possible link between ErbB2 and glucose metabolism. Recent in vivo and in vitro studies observed a role beyond oncogenesis of ErbB2 has been identified with regard to the relationship between ErbB2 and diabetes ${ }^{15}$. The fatty acid synthase (FASN) activity may partly account for the link between ErbB2 and diabetes, because of overexpression of ErbB2 through a phosphatidylinositol 3'-kinase-dependent pathway promotes the expression of FASN ${ }^{25}$. Of importance, studies provided plenty of evidence indicating that circulating FASN is a candidate biomarker of diagnostic and prognostic for diabetes ${ }^{26}$, and it has been associated with insulin action, glucose metabolism and resistance in the development of T2D ${ }^{27}$. A study performed by Fernandez- 'Real et al. ${ }^{28}$ reporting that serum ErbB2 concentrations were positively associated with insulin resistance in obese subjects, indicating that ErbB2 might have a role in the pathophysiology of diabetes ${ }^{28}$. Furthermore, studies provided strong evidence support the notion that higher serum ErbB2 levels may rely on ErbB2 
leads to increase in insulin resistance ${ }^{28}$, and hyperglycemia ${ }^{28,29}$. In agreement with previous study, our data also showed that T2D patients with higher serum ErbB2 concentrations had an increase in insulin resistance in terms of HOMA-IR and Matsuda index compared to those with lower ErbB2 concentrations. However, we did not observe any difference in HOMA-B across quartiles in all recruited subjects.

A significantly increased in serum ErbB2 concentrations along with the progress of the deteriorate glucose metabolism was observed ${ }^{29}$. Ashfaque et al. reported that serum ErbB2 levels were significantly higher in T2D patients compared to those of subjects with impaired fasting glucose (IFG) and/or impaired glucose tolerance (IGT), and IFG and IGT subjects had a higher ErbB2 compared to those with normal glucose tolerance, after adjusting for age, sex, and body mass index ${ }^{29}$. In this study, we did not have data regarding the difference in ErbB2 concentrations in populations with different glucose metabolism conditions. However, we observed that diabetic patients with higher ErbB2 levels had larger GV compared to those with lower serum ErbB2 concentrations.

We also analyzed the relationships between serum ErbB2 concentrations and GV, $\beta$-cell function, and insulin resistance. In agreement with previous study ${ }^{28,29}$, our multivariate linear analysis adjusting for age sex, and BMI indicated that, as expected, patients with higher ErbB2 values had higher HOMA-IR values and decrease in Matsuda index values. We did not observe correlation between ErbB2 values and $\beta$-cell function in the present study. Of most importance, our data showed that serum ErbB2 concentrations were strongly positively correlated with GV (MAGE, SD and CV\%). However, we do not have data for these observation, future studies ascertain the underlining mechanisms of ErbB2 leading to amplify glycemic fluctuations are needed.

Diabetic patients achieving $\mathrm{HbA}_{1 \mathrm{c}}$ in target values is imperative for physicians choosing glucose-lowering agents ${ }^{30}$. However, $\mathrm{HbA}_{1 \mathrm{C}}$ does not provide enough evidence of daily glucose variations ${ }^{31,32}$. Studies demonstrate that glucose variations, especially large MAGE, is the independent risk factor for long-term diabetic complications in T2D ${ }^{31,32}$. The underlying mechanisms may be the reason by acute glucose variations triggering the oxidative stress, which impaired the epithelial cells ${ }^{33}$. Retrospective CGM provides glucose readings at every 5 minutes interval lasting at least for 72 hours, which is an optimal system analyzing GV in diabetic patients ${ }^{34}$. In this study, an increased ErbB2 levels were positively correlated with GV in T2D patients, which indicating that ErbB2 may be a potential treatment target for diabetic patients for improvement in glycemic control.

\section{Conclusion}

Serum ErbB2 concentration was positively correlated to the glycemic variations in newly diagnosed T2D patients. Our data indicating that ErbB2 may be a potential treatment target for diabetic patients for improvement in glycemic control.

\section{Declarations}




\section{Ethics approval and consent to participate}

The study was approved by the ethics committee of Yancheng No. 1 People's Hospital, The Fourth Affiliated Hospital of Nantong University, China. All patients gave written informed consent. The methods were conducted in accordance with the Declaration of Helsinki guidelines, including any relevant details.

\section{Consent for publication}

Written informed consent for publication was obtained from all participants.

\section{Availability of data and materials}

The data sets generated during and/or analyzed during the current study are not publicly available but are available from the corresponding author on reasonable request.

\section{Competing interests}

No competing financial interests exist.

\section{Funding}

This research was funded by Nanjing Public Health Bureau Project (No. YKK11110), and Nanjing Committee of Science and Technology project (No. 201201108).

\section{Authors' contributions}

YQ. S., and YM. L. contributed to the conception and design of the study. LL. J., and Y. H., contributed to the Conduct/data collection. X. H. contributed to data analysis. FF. L. contributed to manuscript writing. YQ. S., and YM. L. final approval of the manuscript.

\section{Acknowledgments}

We appreciated Prof. Lei Ye who contributed to the final proof of the manuscript.

\section{References}

1 Rubin, I. \& Yarden, Y. The basic biology of HER2. Annals of oncology : official journal of the European Society for Medical Oncology 12 Suppl 1, S3-8, doi:10.1093/annonc/12.suppl_1.s3 (2001).

2 Molina, R., Escudero, J. M., Munoz, M., Auge, J. M. \& Filella, X. Circulating levels of HER-2/neu oncoprotein in breast cancer. Clinical chemistry and laboratory medicine 50, 5-21, doi:10.1515/cclm.2011.822 (2012).

3 Iqbal, N. Human Epidermal Growth Factor Receptor 2 (HER2) in Cancers: Overexpression and Therapeutic Implications. Molecular biology international 2014, 852748, doi:10.1155/2014/852748 
(2014).

4 Burstein, H. J. The distinctive nature of HER2-positive breast cancers. The New England journal of medicine 353, 1652-1654, doi:10.1056/NEJMp058197 (2005).

5 Yan, M., Parker, B. A., Schwab, R. \& Kurzrock, R. HER2 aberrations in cancer: implications for therapy. Cancer treatment reviews 40, 770-780, doi:10.1016/j.ctrv.2014.02.008 (2014).

6 Scholl, S., Beuzeboc, P. \& Pouillart, P. Targeting HER2 in other tumor types. Annals of oncology : official journal of the European Society for Medical Oncology 12 Suppl 1, S81-87, doi:10.1093/annonc/12.suppl_1.s81 (2001).

7 Menard, S., Fortis, S., Castiglioni, F., Agresti, R. \& Balsari, A. HER2 as a prognostic factor in breast cancer. Oncology 61 Suppl 2, 67-72, doi:10.1159/000055404 (2001).

8 Giovannucci, E. et al. Diabetes and cancer: a consensus report. CA: a cancer journal for clinicians 60, 207-221, doi:10.3322/caac.20078 (2010).

9 Barone, B. B. et al. Long-term all-cause mortality in cancer patients with preexisting diabetes mellitus: a systematic review and meta-analysis. Jama 300, 2754-2764, doi:10.1001/jama.2008.824 (2008).

10 Rao Kondapally Seshasai, S. et al. Diabetes mellitus, fasting glucose, and risk of cause-specific death. The New England journal of medicine 364, 829-841, doi:10.1056/NEJMoa1008862 (2011).

11 Frasca, F. et al. The role of insulin receptors and IGF-I receptors in cancer and other diseases. Archives of physiology and biochemistry 114, 23-37, doi:10.1080/13813450801969715 (2008).

12 Vazquez-Martin, A., Oliveras-Ferraros, C. \& Menendez, J. A. The antidiabetic drug metformin suppresses HER2 (erbB-2) oncoprotein overexpression via inhibition of the mTOR effector p70S6K1 in human breast carcinoma cells. Cell Cycle 8, 88-96, doi:10.4161/cc.8.1.7499 (2009).

13 Ray, A. Tumor-linked HER2 expression: association with obesity and lipid-related microenvironment. Hormone molecular biology and clinical investigation 32, doi:10.1515/hmbci-20170020 (2017).

14 Ferroni, P. et al. Type 2 Diabetes and Breast Cancer: The Interplay between Impaired Glucose Metabolism and Oxidant Stress. Oxidative medicine and cellular longevity 2015, 183928, doi:10.1155/2015/183928 (2015).

15 Jalving, M. et al. Metformin: taking away the candy for cancer? Eur J Cancer 46, 2369-2380, doi:10.1016/j.ejca.2010.06.012 (2010). 

of Diabetes: A Population-Based Cohort Study. Diabetes care 42, 1582-1588, doi:10.2337/dc18-2556 (2019).

17 Weng, J. et al. Effect of intensive insulin therapy on beta-cell function and glycaemic control in patients with newly diagnosed type 2 diabetes: a multicentre randomised parallel-group trial. Lancet $\mathbf{3 7 1}$, 1753-1760, doi:10.1016/S0140-6736(08)60762-X (2008).

$18 \mathrm{Li}, \mathrm{F}$. F. et al. Features of glycemic variations in drug naive type 2 diabetic patients with different HbA1c values. Scientific reports 7, 1583, doi:10.1038/s41598-017-01719-y (2017).

19 Li, F. F. et al. Influence of Acarbose on Plasma Glucose Fluctuations in Insulin-Treated Patients with Type 2 Diabetes: A Pilot Study. International journal of endocrinology 2015, 903524, doi:10.1155/2015/903524 (2015).

20 Li, F. F. et al. Blood Glucose Fluctuations in Type 2 Diabetes Patients Treated with Multiple Daily Injections. Journal of diabetes research 2016, 1028945, doi:10.1155/2016/1028945 (2016).

21 Zhou, J. et al. Reference values for continuous glucose monitoring in Chinese subjects. Diabetes care 32, 1188-1193, doi:10.2337/dc09-0076 (2009).

22 Matthews, D. R. et al. Homeostasis model assessment: insulin resistance and beta-cell function from fasting plasma glucose and insulin concentrations in man. Diabetologia 28, 412-419 (1985).

23 Matsuda, M. \& DeFronzo, R. A. Insulin sensitivity indices obtained from oral glucose tolerance testing: comparison with the euglycemic insulin clamp. Diabetes care 22, 1462-1470 (1999).

24 Sasaki, R. et al. Association of Waist Circumference and Body Fat Weight with Insulin Resistance in Male Subjects with Normal Body Mass Index and Normal Glucose Tolerance. Intern Med 55, 1425-1432, doi:10.2169/internalmedicine.55.4100 (2016).

25 Kumar-Sinha, C., Ignatoski, K. W., Lippman, M. E., Ethier, S. P. \& Chinnaiyan, A. M. Transcriptome analysis of HER2 reveals a molecular connection to fatty acid synthesis. Cancer research 63, 132-139 (2003).

26 Fernandez-Real, J. M. et al. Extracellular fatty acid synthase: a possible surrogate biomarker of insulin resistance. Diabetes 59, 1506-1511, doi:10.2337/db09-1756 (2010).

27 Menendez, J. A., Vazquez-Martin, A., Ortega, F. J. \& Fernandez-Real, J. M. Fatty acid synthase: association with insulin resistance, type 2 diabetes, and cancer. Clinical chemistry 55, 425-438, doi:10.1373/clinchem.2008.115352 (2009).

28 Fernandez-Real, J. M. et al. Serum HER-2 concentration is associated with insulin resistance and decreases after weight loss. Nutrition \& metabolism 7, 14, doi:10.1186/1743-7075-7-14 (2010). 
29 Memon, A. A. et al. Circulating human epidermal growth factor receptor 2 (HER2) is associated with hyperglycaemia and insulin resistance. Journal of diabetes 7, 369-377, doi:10.1111/17530407.12184 (2015).

30 Inzucchi, S. E. et al. Management of hyperglycemia in type 2 diabetes, 2015: a patient-centered approach: update to a position statement of the American Diabetes Association and the European Association for the Study of Diabetes. Diabetes care 38, 140-149, doi:10.2337/dc14-2441 (2015).

31 Nathan, D. M. et al. Translating the A1C assay into estimated average glucose values. Diabetes care 31, 1473-1478, doi:10.2337/dc08-0545 (2008).

32 Del Prato, S. In search of normoglycaemia in diabetes: controlling postprandial glucose. International journal of obesity and related metabolic disorders : journal of the International Association for the Study of Obesity 26 Suppl 3, S9-17, doi:10.1038/sj.ijo.0802172 (2002).

33 Monnier, L. et al. Activation of oxidative stress by acute glucose fluctuations compared with sustained chronic hyperglycemia in patients with type 2 diabetes. Jama 295, 1681-1687, doi:10.1001/jama.295.14.1681 (2006).

34 Rodbard, D. et al. Improved quality of glycemic control and reduced glycemic variability with use of continuous glucose monitoring. Diabetes technology \& therapeutics 11, 717-723, doi:10.1089/dia.2009.0077 (2009). 